\title{
THERMALLY INDUCED REVERSIBLE EASY AXIS ROTATIONS IN PERMALLOY THIN FILMS
}

\author{
WINFRIED H. G. HORSTHUIS*
}

Centre for Microelectronics Twente, Sensor Group, P.O. Box 545, 7500 AM Enschede (The Netherlands) KEES J. M. EIJKEL AND RENÉ M. DE RIDDER

University of Twente, Department of Electrical and Electronic Engineering, P.O. Box 217, 7500 AE Enschede (The Netherlands)

HENK LEEUWIS

Centre for Microelectronics Twente, Sensor Group, P.O. Box 545, 7500 AM Enschede (The Netherlands) (Received November 17, 1987; revised March 1, 1988; accepted April 11, 1988)

The thermal stability of the easy axis orientation in thin Permalloy films was studied. This orientation was measured by two methods, i.e. the magneto-optic Kerr effect and a novel measurement scheme based on magnetoresistive effects. It appeared that even for small temperature changes rotations of the easy axis occurred. Together with the commonly observed irreversible rotations (thermal annealing), reversible rotations were also measured. Variation of film composition, substrate layer and different annealing steps were tried to obtain a better understanding of this unexpected behaviour. No well-defined correlation could be established between the varied parameters and thermal behaviour.

\section{INTRODUCTION}

Permalloy is a well-known material frequently used in magnetic sensors. In some applications the operation of the device is based on the uniaxial anisotropy of the Permalloy film. The orientation of the easy axis of the film is important for the performance of such devices ${ }^{1-4}$

In previously published papers ${ }^{5,6}$ it was shown that the easy axis orientation is susceptible to temperature changes. These studies concerned irreversible rotations of the easy axis. It was usually indicated that annealing of the films in the presence of a strong magnetic field results in a temperature-independent easy axis orientation up to the annealing temperature.

We carried out some experiments on Permalloy films to study the stability of the easy axis orientation under the influence of small temperature changes. It was observed that, even in the case of annealed films, minor rotations occurred which were largely reversible. Substrate, film composition and annealing steps were varied to find out whether this effect could be eliminated.

\footnotetext{
* Present address: Akzo Research B.V., Applied Physics Department, Velperweg 76, 6824 BM Arnhem, The Netherlands. 


\section{EXPERIMENTS}

Permalloy films of $50 \mathrm{~nm}$ thickness were deposited on oxidized silicon wafers by either electron gun vacuum evaporation or r.f. sputtering. Deposition parameters are listed in Table I. The magnetic properties of sputtered and evaporated films were highly comparable.

TABLE I

PERMALLOY DEPOSITION PARAMETERS

\begin{tabular}{ll}
\hline R.f. sputtering & Swept electron gun evaporation \\
\hline Target, $\mathrm{Ni}_{82} \mathrm{Fe}_{18}$ & Sources, $\mathrm{Ni}$ and $\mathrm{Fe}$ \\
Water-cooled substrate holder; Sputter-gas, Ar & Substrate temperature $200^{\circ} \mathrm{C}$ \\
Residual pressure, $<2 \times 10^{-5} \mathrm{~Pa}$ & Residual pressure, $5 \times 10^{-6} \mathrm{~Pa}$ \\
Sputter pressure, $2.5 \mathrm{~Pa}$ & Evaporation pressure, $\leqslant 10^{-5} \mathrm{~Pa}$ \\
Rate, $0.17 \mathrm{~nm} \mathrm{~s}^{-1}$ & Rate, $0.3 \mathrm{~nm} \mathrm{~s}^{-1}$ \\
\hline
\end{tabular}

Two measurement methods were used to study the temperature effects: magneto-optic Kerr measurements and a novel measurement scheme developed by Eijkel et al. ${ }^{7}$

\subsection{Kerr measurements}

From the longitudinal magneto-optic Kerr effect, the component of the magnetization parallel to the plane of incidence of a (laser) light beam in a thin Permalloy film can be determined.

A pair of Helmholtz coils is used to generate an in-plane sinusoidal saturating field of about $1000 \mathrm{~A} \mathrm{~m}^{-1}$ perpendicular to the easy axis of the Permalloy film. A signal proportional to this field is applied to the horizontal channel of an oscilloscope, while the Kerr signal, which in our configuration is a measure for the magnetization component in the plane of the film and parallel to the easy axis, is fed to the vertical channel.

The $M v$ s. $H$ curve thus obtained is strongly influenced by the orientation of the easy axis with respect to the applied field. If the excitation field is perpendicular to the easy axis, the oscilloscope shows a straight line. In all other cases, as a result of the rotation of part of the magnetization, a difference between the responses in increasing and decreasing excitation fields occurs, resulting in a loop as depicted in Fig. 1.

Both films of large area ( 2 in diameter) and small chips $(5 \mathrm{~mm} \times 5 \mathrm{~mm}$ ) could be mounted in the equipment. The spot size $S$ (diameter) of the light beam (He-Ne laser) could be varied in the range $0.1 \mathrm{~mm}<S<10 \mathrm{~mm}$. The films were heated by means of focused light from a projection bulb, enabling the temperature of the Permalloy to be raised from room temperature to a maximum of approximately $90^{\circ} \mathrm{C}$.

The experiments were carried out as follows. First the films were aligned with the field of the Helmholtz coils. Then they were heated, while the Kerr response was observed. After stabilization of the response, the lamp was switched off, and the 


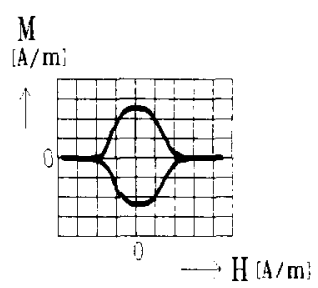

Fig. 1. Oscillogram representing the magnetization component $M$ in the plane of the film and parallel to the easy axis $v s$. the applied field $H$ for a slight misalignment between easy axis and applied field.

response was again monitored. Both reversible and irreversible rotations of the easy axis were observed. Alternatively, the films were re-aligned with respect to the excitation field during heating, which results in a continued rotation of the easy axis. Figures 2 and 3 show some typical responses measured.

Maximum rotations up to $6^{\circ}$ were measured in the case of the repeated alignment method. With a single alignment cycle the rotation was limited to less than $1^{\circ}$. In Figs. 2 and 3 it is clearly visible that the major part of the rotation is reversible; only a minor rotation of about $0.1^{\circ}$ remains. It appeared that the effect greatly depended on the laser spot size. If a small spot (less than $1 \mathrm{~mm}$ in diameter) was used, the effect varied strongly with the position of the spot on the film. If, in contrast, a spot diameter in excess of $3 \mathrm{~mm}$ was used, the response was independent of the location on the film. Apparently the easy axis rotations depend on local film properties. In case of a large spot size, the effect of many different areas is averaged. No noticeable differences were observed between sputtered and evaporated films.
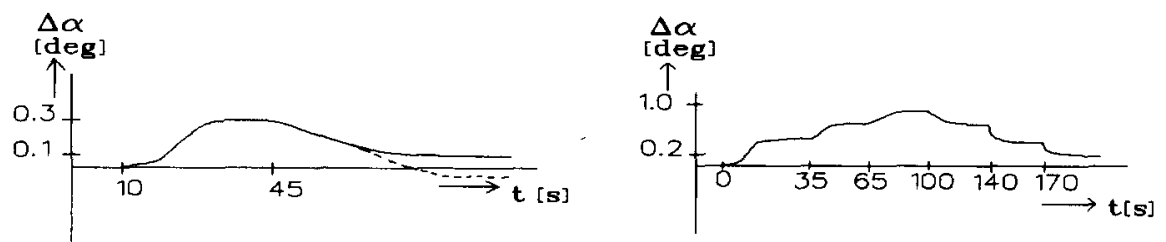

Fig. 2. $\Delta \alpha$ (rotation of the easy axis) $v s$. time. After $10 \mathrm{~s}$ the heating lamp is switched on, and after $45 \mathrm{~s}$ it is switched off again (maximum temperature of sample, approximately $70^{\circ} \mathrm{C}$ ): - - , most common response; ---, response found in some cases.

Fig. 3. $\Delta \alpha v$ s. time. The lamp is switched on at $t=0 \mathrm{~s}$. After 35 and $65 \mathrm{~s}$ the easy axis is mechanically realigned with the applied field. After $100 \mathrm{~s}$ the lamp is switched off. The maximum temperature is about $80^{\circ} \mathrm{C}$. After 140 and $170 \mathrm{~s}$ the easy axis is aligned with the applied field again.

To our knowledge, this is the first time that such significant reversible rotations have been reported for low temperature changes in films produced by conventional deposition techniques. Some variation in film parameters has been tried in order to obtain a better understanding of the effect.

Since it was thought that film-substrate stresses could play an important role owing to magnetostriction effects, the first variation tried was a change in substrate material. A $2 \mu \mathrm{m}$ thick polyimide intermediate layer was introduced to obtain a second substrate material with definitely different mechanical properties. Measure- 
ments indicated little influence of the substrate. Only the effect of the repeated alignment changed significantly. In contrast with Permalloy deposited on silica, the films on polyimide showed a less pronounced continued rotation after re-alignment with respect to the excitation field. Maximum obtainable rotations were now of the order of $3^{\circ}$.

A second variation concerned the composition of the evaporated films. We tried films with a nickel content varying from 80.2 to 86.7 at. $\%$. A nickel content of $82 \%$ is usually considered to be magnetostriction free ${ }^{8}$. It appeared that the easy axis rotations occurred for all film compositions. The strongest rotations were found for compositions with a high nickel content.

The third variation consisted of film annealing to examine whether the easy axis rotations could be eliminated. The Permalloy films were annealed in a Pyrex tube flushed with heated dry nitrogen. The tube was placed in a solenoid coil to apply a magnetic field of $10 \mathrm{kA} \mathrm{m}^{-1}$ parallel to the easy axis of the film. Only films of size $5 \mathrm{~mm} \times 5 \mathrm{~mm}$ could be mounted in the tube. The temperature of the film was monitored with a thermocouple. The maximum obtainable temperature was $350^{\circ} \mathrm{C}$.

The magnetic properties (coercivity $H_{\mathrm{c}}$, anistropy field $H_{\mathrm{k}}$ and saturated magnetic moment $m_{\mathrm{s}}$ ) of the films were determined by means of inductive hysteresis measurements before and after the annealing step. After annealing at $200^{\circ} \mathrm{C}$ for $1 \mathrm{~h}$ no effect on these film properties could be determined. The sensitivity of the easy axis orientation to temperature changes did not significantly change either. For temperatures in excess of $220^{\circ} \mathrm{C}$ the (uncoated) films degraded visibly owing to oxidation, causing considerable changes in magnetic properties.

In order to check the annealing conditions, some films were annealed at the same temperatures and duration, mounted with the easy axis perpendicular to the solenoid field. The anisotropy constant in these films decreased, as expected from ref. 5. Table II shows some measured magnetic properties $\left(H_{\mathrm{c}}, H_{\mathrm{k}}\right.$ and $\left.m_{\mathrm{s}}\right)$ of samples before and after annealing. Annealing for long periods at high temperature resulted in both a decreasing $m_{\mathrm{s}}$ and an increased coercivity. Both effects are attributed to film surface oxidation. The decrease in $m_{\mathrm{s}}$ stems from the decrease in magnetic volume, whereas the increased coercivity can be ascribed to induced imperfections such as an irregular oxidation depth and consequently an irregular magnetic surface layer.

Finally, a preliminary experiment was carried out on a relatively thick Permalloy film $(200 \mathrm{~nm}$ instead of the usual $50 \mathrm{~nm})$. The influence of temperature variations on this film appeared to be considerably smaller. A tentative assumption following from this result is that the internal film stress in the region between the nucleation layer and the "bulk" of the film is responsible for the observed effects. For thicker films, the contribution of this region becomes less dominant. Unfortunately, such thick films have their drawbacks for application in sensor concepts such as those in ref. 1 . The influence of film thickness on the thermally induced easy axis rotations will be studied in the near future.

\subsection{Experiments based on the magnetoresistive effect}

The magneto-optic Kerr measurements are indicative, but no accurate data on the easy axis rotations can be obtained with our equipment. It was thought useful to 


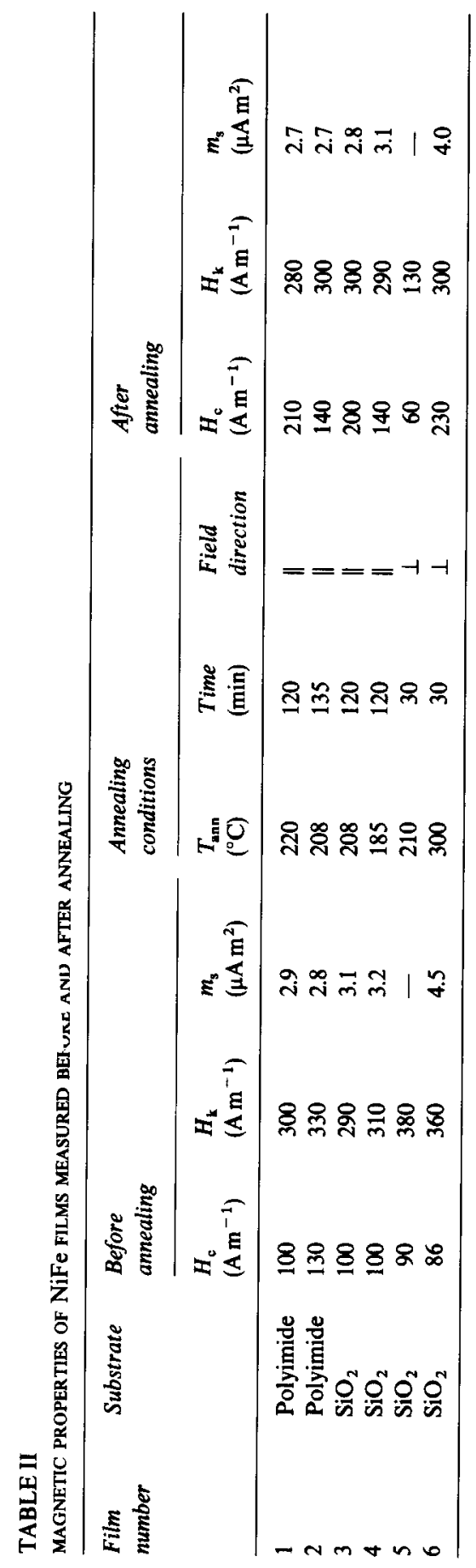


study the effects with a second measuring technique. The second technique used, based on the planar Hall effect, is described in detail elsewhere ${ }^{7}$, and can provide accurate quantitative results. This technique is briefly discussed below.

An a.c. magnetic field $(90 \mathrm{~Hz})$ is superimposed on a d.c. field, care being taken to ensure that the field strength is always greater than the anisotropy field in the Permalloy to prevent switching in the film. When the easy axis of the Permalloy is either parallel or perpendicular to the field, no rotation of magnetization occurs. In all other cases, the magnetization in the Permalloy will rotate from a direction closer to the easy axis to a direction closer to the field and back. This oscillation in magnetization direction can be detected using the planar Hall effect. Four electrical contacts have to be positioned on the Permalloy, two for the current through the sample, and two for the planar Hall voltage. These contacts were realized either by depositing the Permalloy onto an aluminium electrode pattern or by the deposition of an Mo-Al double layer on top of the Permalloy. The use of a molybdenum interlayer proved necessary to ensure a stable contact.

The signal voltage from the film is amplified with a lock-in amplifier (LIA). The easy axis of the Permalloy can be positioned either parallel or perpendicular to the field resulting in a zero output signal of the LIA. Rotation of the easy axis over a small angle $\delta$ will give rise to a non-zero output signal, linearly dependent on $\delta$.

The Permalloy film is heated with hot air from a blower, covering a temperature range from 20 to $80^{\circ} \mathrm{C}$. To obtain an indication of the temperature of the Permalloy, the resistivity of the Permalloy is measured. From earlier measurements we found that resistivity is a linear function of temperature for a typical Permalloy film in the temperature range mentioned above ${ }^{7}$. The temperature of the Permalloy can be derived from the measured value of the resistivity with a maximum error of $1{ }^{\circ} \mathrm{C}$. The temperature signal and the output signal of the LIA are connected to an $X-Y$ recorder to produce direct easy axis orientation $v$ s. temperature plots.

To determine the mechanical stability of the equipment during heating the position of a laser beam, reflected on a mirror glued onto the Permalloy, was monitored. In the temperature range indicated above no mechanical rotation of the film was observed. A few typical results are shown in Figs. 4-6.

The measurements start at point $A$. It is clear that a reversible and an irreversible effect are present. The irreversible effect appears above a certain temperature (Fig. 4). When the Permalloy has been subjected to a higher temperature $T_{\mathrm{a}}$ for some time, the irreversible effect disappears at temperatures below $T_{\mathrm{a}}$, and

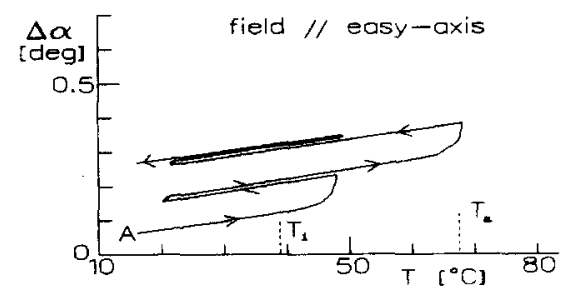

Fig. 4. A typical measurement using the magnetoresistive effect. From point $A$ the temperature was raised. A reversible effect is observed. Above $T_{1}$ an irreversible effect appears. When the film is subjected to a higher temperature $T_{\mathrm{a}}$ from some time, the irreversible effect disappears at lower temperatures. 
only the reversible effect remains. When, after the disappearance of the irreversible effect, the easy axis is manually re-oriented perpendicular to the field, an irreversible effect is again apparent at much lower temperatures (Fig. 5). In general, the measurements were hard to interpret, because of pronounced differences between different films, especially with respect to the irreversible effect. Films on polyimide showed similar behaviour to the films on silica, but behaved less predictably with respect to the irreversible effect.

Measurements were performed also on a commercially available device (Philips KMZ 10a). Only the galvanomagnetic measurement technique can be used for encapsulated devices. This device consists of a Wheatstone bridge of four Permalloy magnetoresistors with barberpoles ${ }^{9}$. Intrinsic anisotropy of the material combines with the form effect in the permalloy strips to give an effective anisotropy ${ }^{10}$. The rotation of the effective anisotropy is measured. Figure 6 shows the measured results from this device, where a reversible effect is again apparent. In the temperature range used, no irreversible effect was seen. To our knowledge, these devices are stabilized by thermal annealing at a much higher temperature than the temperature range for normal use.
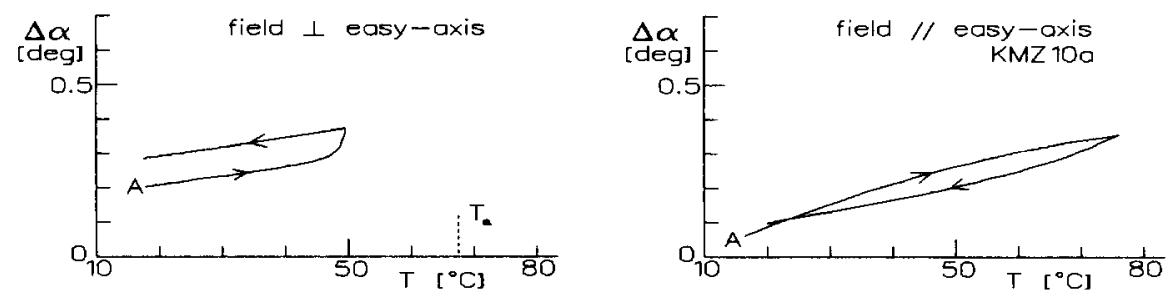

Fig. 5. Immediately after the measurement shown in Fig. 4, the film was positioned with its easy axis perpendicular to the applied field. The irreversible effect now appears at lower temperatures.

Fig. 6. The observed reversible rotation of the easy axis with temperature also occurs in commercial devices. Depicted here is a measurement on a Philips KMZ 10a magnetoresistive device.

\section{CONCLUSIONS}

Measurements have been performed to determine the stability of the easy axis orientation in thin Permalloy films during small temperature changes. This orientation appears to be temperature dependent. Reversible rotations in excess of $1^{\circ}$, as well as small irreversible rotations, occur even for small temperature changes of the order of $20^{\circ} \mathrm{C}$.

Stress could play an important role in this phenomenon. However, films deposited on both silica and polyimide showed these effects, despite the difference in mechanical properties of these two substrate materials.

Variation in film composition had only a minor influence.

An indication has been obtained that an increased film thickness reduces the observed effect. More measurements must be carried out to confirm the influence of film thickness.

It is concluded that devices based on the easy axis orientation in thin Permalloy films are sensitive to changes in ambient temperature. 


\section{ACKNOWLEDGMENTS}

The authors wish to thank Poul de Haan for his assistance in the Kerr measurements and many fruitful discussions.

This work was supported by the Centre for Microelectronics Twente and the Netherlands Technology Foundation.

\section{REFERENCES}

I R. M. de Ridder and J. H. Fluitman, Orthogonal thin film magnetometer using the anisotropic magnetoresistance effect, IEEE Trans. Magn., 20 (1984) 690-692.

2 G. Reiniger, Drehwinkelmessung mit Magnetfeldsensoren, Elektronik, 23 (1986) 129-136.

3 G. R. Hoffman, J. K. Birtwistle and E. W. Hill, The performance of magnetoresistive vector magnetometers with optimised conductor and anisotropy axis angles, IEEE Trans. Magn., 19 (1983) $2139-2141$.

4 S. Tumanski and M. M. Stabrowski, Optimization of the performance of a thin film permalloy magnetoresistive sensor, IEEE Trans. Magn., 20 (1984) 963-965.

5 W. Metzdorf, Die Beeinfluszbarkeit der uniaxialen magnetischen Anisotropie aufgedampfter Permalloyschichten durch Tempern im Magnetfeld, Z. Angew. Phys., 18 (1964) 534-540.

6 M. Takayasu, S. Uchiyama, K. Takahashi and T. Fujii, Isothermal annealing behaviour of zeromagnetostrictive permalloy films, II, experimental, IEEE Trans. Magn., 10 (1974) 552-555.

7 K. J. Eijkel, R. M. de Ridder and P. de Haan, Techniques for measuring small changes in the orientation of the easy-axis in permalloy films, Rev. Sci. Instrum., to be published.

8 Magnetostriktion, in Landolt-Börnstein, Group II, Vol. 9, Springer, Berlin, 1962, pp. 1-123-1-137.

9 U. Dibbern and A. Petersen, The magnetoresistive sensor - a sensitive device for detecting magnetic field variations, Philips Electron. Compon. Appl., 5 (3) (1983) 148-153.

$10 \mathrm{~J}$. H. J. Fluitman, The influence of sample geometry on the magnetoresistance of NiFe films, Thin Solid Films, 16 (1973) 269-276. 